\title{
Bebês com Sequência de Pierre Robin: saúde mental materna e interação mãe-bebê
}

\author{
Infants with Pierre Robin Sequence: Maternal mental \\ health and mother-infant interaction
}

\author{
Camila Guedes de Azevedo NARDI ${ }^{1}$ \\ Olga Maria Piazentin Rolim RODRIGUES ${ }^{2}$ \\ Lígia Ebner MELCHIORI² \\ Manoel Henrique SALGADO3 \\ Lilian D'Aquino TAVANO ${ }^{1}$
}

\begin{abstract}
Resumo
Este estudo pretendeu avaliar o estresse e ansiedade materna, bem como a qualidade da interação de dez mães e seus bebês, de zero a três meses, com Sequência de Pierre Robin, internados em um hospital universitário. Utilizou-se uma escala e um inventário para avaliação do estresse e da ansiedade maternos e um protocolo para avaliação da filmagem da interação mãe-bebê. Os resultados apontaram altos níveis de estresse e ansiedade maternos. Foi encontrada uma relação inversa estatisticamente significativa: quanto maior a ansiedade e o estresse materno, menor a sintonia de interação da díade mãe-bebê. Os resultados indicam a necessidade de um trabalho que minimize a ansiedade e o estresse de mães com bebês internados, favorecendo a interação.
\end{abstract}

Palavras-chave: Ansiedade materna; Estresse materno; Relações mãe-criança.

\begin{abstract}
The aim of this study was to evaluate maternal stress and anxiety and the quality of interaction of 10 mothers and their 0-3-month-old babies with Pierre Robin Sequence hospitalized in a university hospital. We used the Inventory of Stress Symptoms for Adults to evaluate stress and the State-Trait Anxiety Inventory to assess anxiety of the mothers, and a protocol to assess the recording of the mother-infant interaction. The results showed high levels of maternal stress and anxiety. A statistically significant inverse relationship was found: the higher the maternal anxiety and stress, the lower was the mother-infant interaction. The results indicate that work needs to be done to minimize the anxiety and stress of mothers with inpatient babies in order to encourage interaction.
\end{abstract}

Keywords: Anxiety; Stress; Mother-child relations.

2 Universidade Estadual Paulista Júlio de Mesquita Filho, Faculdade de Ciências, Departamento de Psicologia. Av. Edmundo Carrijo Coube, 14-01, 17033-360, Bauru, SP, Brasil. Correspondência para/Correspondence to: O.M.P.R. RODRIGUES. E-mail: <olgarolim@fc.unesp.br>

3 Universidade Estadual Paulista Júlio de Mesquita Filho, Faculdade de Engenharia, Departamento de Engenharia de Produção. Bauru, SP, Brasil.
} 
A Sequência de Pierre Robin (SR) é descrita como "uma tríade de anomalias caracterizada por micrognatia, glossoptose e fissura de palato. Apesar da fissura de palato estar presente na maioria dos casos, pode estar ausente em alguns" (Marques, Souza, Barbieri, \& Bettiol, 2005, p.14) e pode ocorrer "de 1 para 8500 a 1 para 20000 nascimentos" (Fuzza \& Abuabara, 2010, p.233). Para ser considerada como SR deve ser diagnosticada a presença de pelo menos duas das anomalias citadas (Sato, Setten, Sverzut, Moraes, \& Moreira, 2007).

Clinicamente, esse complexo de anomalias se caracteriza por obstrução das vias aéreas e dificuldades na alimentação, principalmente no período neonatal (Marques et al., 2005; Sato et al., 2007). A SR não representa nenhuma síndrome específica, configurando-se como um conjunto de malformações que pode ou não estar associado a outras deformidades do desenvolvimento, sendo a hereditariedade um fator relevante no desenvolvimento desse quadro (Marques et al., 2005; Sato et al., 2007). Fuzza e Abuabara (2010) destacam que, devido à obstrução aérea e a dificuldades alimentares, as crianças com SR apresentam alto risco de desenvolver uma hipóxia crônica, com as complicações subsequentes: falência cardíaca direita, episódios de cianose, hipóxia cerebral, asfixia grave ou mesmo fatal, aumento do trabalho respiratório causado pela fenda palatina, obstrução pela língua e má deglutição, levando a uma falência do crescimento. Todavia, os autores destacam que, apesar de constituir inicialmente uma emergência médica em razão da obstrução respiratória, clinicamente, observa-se melhora gradual na respiração e alimentação nos primeiros meses ou anos de vida, comprovada por meio de análises cefalométricas que mostram o crescimento mandibular. Dessa forma, a SR é uma das poucas deformidades que melhora com a idade.

O nascimento de uma criança que necessita de cuidados intensivos em um hospital configura-se um evento estressor, causando na família uma situação inesperada e geradora de ansiedade. Envolvidos no contexto de hospitalização, os familiares vivenciam sensações e sentimentos desconhecidos, que podem vir acompanhados da necessidade de separação temporária do bebê e da incerteza quanto a sua sobrevivência (Padovani, Linhares, Carvalho, Duarte, \& Martinez, 2004).

A família é atingida emocionalmente com o nascimento de uma criança com SR e, consequentemente, com a sua hospitalização, sem contar que a falta de informação sobre essa malformação e a presença de fissura de lábio e/ou palato acentuam ainda mais o impacto emocional. Segundo Perosa, Silveira e Canavez (2008), a vulnerabilidade biológica desses recém-nascidos agrava a dificuldade de adaptação dos genitores, pois o contato com a mãe é restrito quando da internação, e a separação potencializa o sentimento de impotência em oferecer cuidados adequados ao filho. Baldini e Krebs (2005) afirmam que a internação de uma criança em estado grave pode gerar uma situação de crise psicológica para o paciente e sua família.

Carvalho (2005) destaca que mães com bebês internados podem apresentar percepções distorcidas sobre a evolução do filho, subestimando ou superestimando a evolução do quadro clínico. Esses sentimentos podem ser atenuados ou reforçados de acordo com a oportunidade que a mãe tem ou não de participar, de alguma forma, dos cuidados de seu filho (Scochi, Kokuday, Riul, Rossanez, Fonseca, \& Leite, 2003).

Um dos pontos defendidos na humanização hospitalar é a participação ativa dos pais no processo de recuperação do filho internado, favorecendo a formação e fortalecimento do vínculo afetivo (Reichert, Lins, \& Collet, 2007). Por essa razão, a presença dos genitores, ou pelo menos das mães, nas Unidades de Terapia Intensiva Neonatal (UTIN), está baseada na preocupação com o vínculo afetivo que precisa ser estabelecido entre o bebê e seus principais cuidadores (Ainsworth, Blehar, Waters, \& Wall, 1978; Bowlby, 1990).

Mendes (2007) considera que a interação precoce desenvolvida entre a díade mãe-bebê é essencial para a construção da vida psíquica do ser humano, sendo que o vínculo materno, definido a partir da comunicação da mãe com o bebê, exerce um papel fundamental nesse processo. A criança passa a identificá-la através de estímulos perceptuais, tais como o sorriso, o contato visual, as vocalizações e as expressões faciais, além de ter 
grande parte de suas necessidades supridas por intervenção do cuidador. Por outro lado, o bebê também participa dessa relação, emitindo sinais como o choro, balbucios, sorrisos, reflexo de agarrar, agitação ou mesmo engatinhar ou andar em direção à mãe, dependendo de sua capacidade motora (Bolwby, 1990).

O ideal é que desde cedo a mãe entre em um estado de sensibilidade e procure se colocar no lugar do bebê, adaptando-se às necessidades dele, transformando-se em um ser responsável pelo seu desenvolvimento emocional (Souza, 2005). Ainsworth et al. (1978) definiram a sensibilidade materna como decorrente: a) da habilidade da mãe para reconhecer e decodificar os sinais de comunicação emitidos pelo bebê; b) da rapidez das respostas dadas a ele (sincronia) frente ao que ela decodificou, e c) da adequação das respostas do socializador e da sua coerência diante das necessidades da prole. Em função de como a mãe reage aos sinais emitidos pelo bebê, o relacionamento entre eles será mais ou menos prazeroso, estabelecendo o vínculo em um processo de trocas bidirecionais.

Segundo Alfaya e Schermann (2005) e lungano (2009), a qualidade da relação estabelecida entre mãe e bebê se inicia por meio de comportamentos que promovem a interação da díade, incluindo desde o amamentar até o brincar. A construção desses comportamentos interativos geralmente está relacionada aos padrões construídos pela mãe, na sua infância, com sua própria mãe e outros cuidadores principais. Desse modo, na medida em que tais comportamentos começam a ser repetidos, a criança apresenta um padrão de interação social, construindo a representação de si mesma e do ambiente (Bolwby, 1990).

O nascimento de um bebê que necessita de cuidados especiais afeta a interação com a mãe, pois traz consequências tanto para a vivência dela, como para a organização das condutas do bebê (Camarneiro, Alves, Ferreira, \& Gomes, 2009; Andreani, Custódio, \& Crepaldi, 2006). De acordo com Alfaya e Shermann (2005), além dos agravos clínicos enfrentados pelo recém-nascido, são observadas dificuldades na interação dos pais com o bebê durante a hospitalização, e tais limitações podem prejudicar o desenvolvimento da sensibilidade materna e, consequentemente, do estabelecimento do vínculo de apego entre eles.

O período pós-natal é uma época em que as percepções maternas e os padrões interacionais com seus bebês hospitalizados estão em formação. A maneira como a mãe interage com o bebê irá, em parte, influenciar o comportamento dele em relação a ela, além de que a situação desconfortável da hospitalização, envolvendo dor, tubos e sondas, grande luminosidade, excesso de ruído, entre outros, vão, também, influenciar o comportamento do bebê, podendo comprometer ou dificultar essa reciprocidade (Feijó, 1998; Lira, 2004; Padovani et al., 2004; Mendes, 2007; Scochi et al., 2003).

Wust e Viera (2011) realizaram uma pesquisa qualitativa com o objetivo de descrever o relacionamento mãe-filho pré-termo após a alta da unidade de terapia intensiva neonatal, considerando o fato de que ambos passaram por privação de contato após o parto. Participaram quatro mães cujos filhos estiveram hospitalizados por três meses, sendo os dados coletados por meio de entrevista em visitas domiciliares, na primeira semana após a alta hospitalar. Os resultados apontaram várias dificuldades vivenciadas pelas mães devido à crise inicial provocada pela separação logo após o nascimento, assim como a interação prejudicada pelo pouco ou nenhum contato com o filho durante o período de hospitalização. Emergiram nesse estudo aspectos que são considerados comuns às mães de recém-nascidos pré-termo, tais como sentimento de fuga, medo, negação e dificuldade ao ver o filho submetido ao tratamento, tão frágil e conectado a equipamentos. Sendo assim, segundo as autoras, todos esses fatores associados podem, inicialmente, comprometer o vínculo entre mãe e filho; porém, os estágios pelos quais os pais passam para a formação do apego acontecem de forma diferenciada, e eles precisam lidar com os diversos sentimentos que emergem nessa situação.

Preocupados com a questão da afetividade na interação mãe-bebê e conscientes da escassez de estudos que contemplem esse aspecto da interação, Seidl-de-Moura et al. (2008) analisaram episódios de interação mãe-bebê em duas faixas 
etárias: bebês com um e cinco meses de idade. Participaram do estudo 56 díades, que foram filmadas em suas casas. As autoras ponderam que seus resultados confirmaram a literatura da área quanto à possibilidade de trocas afetivas em etapas iniciais do desenvolvimento, identificando que as interações face a face são mais frequentes em bebês de um mês de idade, enquanto as atividades mais complexas, como a estimulação por objetos, são mais comuns nas díades com bebês de cinco meses. Os mais novos, conforme já esperado, sorriram muito pouco. As mães dos dois grupos sorriram para seus bebês e apresentaram comportamentos afetivos nas interações.

Em relação à interação mãe-bebê em situação hospitalar, Alfaya e Shermann (2005) avaliaram a sensibilidade materna em três grupos de bebês. Participaram 20 díades com recém-nascidos pré-termo (Grupo 1-G1), 14 díades com recém-nascidos a termo que necessitavam de cuidados intensivos (G2) e 24 díades com recém-nascidos a termo sadios (G3). A interação mãe-bebê foi filmada e depois avaliada, de acordo com um protocolo de avaliação de interação mãe-bebê. Os resultados mostraram que houve maior incidência de sensibilidade materna positiva no G3, indicando que ela foi mais frequentemente identificada em mães de bebês nascidos a termo e sadios.

No caso de bebês com malformações, o nascimento de uma criança de risco produz um impacto na representação materna de um bebê sadio e normal, promovendo um campo de luto e criando a necessidade de reconstrução da imagem que a mãe antecipou durante a gravidez (Perosa et al., 2008; Brum \& Schermann, 2004). Assim, o nascimento inesperado de um bebê em situação de risco faz com que os pais entrem em um estágio de luto tão intenso como a morte real de uma criança, experimentando sentimentos de choque, negação, raiva, culpa, depressão, desesperança, impotência, perda, isolamento, confusão e ansiedade (Castro \& Piccinini, 2004; Mendes, 2007; Baldini \& Krebs, 2005).

O processo de luto pelo bebê idealizado faz com que os pais sintam dificuldade na aceitação dessa criança, tornando mais demorado o processo

132 de ligação emocional da mãe e de outros cuidadores principais com o bebê, uma vez que há uma demora na reconstrução da imagem do bebê real, bem como para se organizarem internamente (Baldini \& Krebs, 2005). Ainda que esses sentimentos sejam muito comuns entre os genitores nessa condição, eles devem passar com o tempo. Brum e Schermann (2004) pontuam que o luto, apesar de doloroso, tem um papel significativo na saúde emocional e no bem-estar da criança.

Perosa et al. (2008) compararam as condições emocionais de 17 mães, cujos filhos nasceram com malformações visíveis, logo após o nascimento e três meses após a alta hospitalar. Os resultados indicaram altos índices de ansiedade e depressão puerperal no pós-parto imediato, ao passo que, três meses após a alta, foi observada uma redução dos sintomas de depressão e dos sinais clínicos de ansiedade.

Em estudo com mães de bebês prematuros hospitalizados, Dantas, Araújo, Paulino e Maia (2012) concluíram que os sintomas depressivos maternos estão inversamente associados à sua rede de apoio social, ou seja, quanto maior o reconhecimento de apoio recebido pelas mães, menor a sintomatologia depressiva, sendo o inverso também verdadeiro. Investigando as variáveis emocionais em mães com bebês prematuros, Fraga, Linhares, Carvalho e Martinez (2008) verificaram que as mais ansiosas podem ser mais atentas para estimular suas crianças. Porém, o excesso da ansiedade pode acarretar dificuldades na experiência da criança em relação à aquisição de autonomia e à iniciativa para explorar o ambiente, uma vez que essas mães podem interagir com os filhos de forma intrusiva e restritiva, dificultando suas aquisições.

Mães com crianças que necessitam de cuidados especiais passam a apresentar problemas para dormir, hipertensão e palpitações, depois da descoberta da enfermidade do filho (Castro \& Piccinini, 2004). Carvalho (2005) comparou os indicadores emocionais maternos e o desenvolvimento dos neonatos pré-termos internados em UTIN, em grupos diferenciados quanto à presença (G1) ou ausência (G2) de sintomas de ansiedade e depressão materna no período neonatal. Os resultados indicaram que no G1 houve potencialização dos sinto- 
mas de ansiedade e depressão durante e após o período de internação. Segundo Padovani et al. (2004), mães que acompanham os filhos internados costumam apresentar altos níveis de ansiedade e depressão, desencadeando sentimentos de apoio emocional inadequado ao bebê. Outros estudos têm indicado que a ansiedade materna traz prejuízos ao desenvolvimento emocional da criança (Linhares, Carvalho, Machado, \& Martinez, 2003; Fraga et al., 2008; Correia \& Linhares, 2007).

A ansiedade é um estado emocional com componentes psicológicos e fisiológicos, comum à experiência humana, sendo propulsora do desempenho (Rey, 2005). No entanto, ela passa a ser patológica quando não existe um objeto específico a que se direcione ou quando é desproporcional à situação que a desencadeia (Andrade \& Gorenstein, 1998). Embora a ansiedade tenha sido investigada em pesquisas que avaliam a interação ou a influência dessa sintomatologia no desenvolvimento de bebês, um conceito praticamente ausente dessas pesquisas é o estresse. Segundo Lipp e Romano (1987), o estresse é uma reação do organismo frente a situações extremamente difíceis ou excitantes que provocam alterações psicológicas, físicas e químicas. Apesar de sua importância, o impacto do estresse não tem sido investigado quando se trata do nascimento de bebês com anomalias. Nesse sentido, o presente estudo pretende avaliar o estresse materno nessas condições.

No Hospital de Reabilitação de Anomalias Craniofaciais de Bauru da Universidade de São Paulo (HRAC/USP), os bebês com SR vêm encaminhados de hospitais do Brasil inteiro, geralmente entre três e dez dias de vida, e permanecem internados na Unidade de Cuidados Especiais (UCE). Na UCE as mães são responsáveis pelos cuidados diários do bebê, sob orientação e supervisão direta dos profissionais da equipe. A investigação da relação entre ansiedade e estresse dessas mães e a interação estabelecida entre elas e o filho poderá contribuir com o trabalho já desenvolvido nessa unidade e em outras semelhantes, não só ampliando os cuidados prestados à saúde da criança, mas também fornecendo dados para a implantação de estratégias de intervenção que facilitem o estabelecimento do vínculo mãe-bebê. O presente estudo pretendeu descrever os níveis de ansiedade e de estresse de mães de bebês com SR e relacioná-los com a qualidade da interação entre ambos.

\section{Método}

\section{Participantes}

Participaram do estudo 10 díades mãe-bebê. Os bebês apresentavam a Sequência de Robin e estavam internados no HRAC/USP, com tempo de internação de 10 a 40 dias (Média - $M=19$ dias). Tinham entre um e 3 meses e 12 dias de idade, sendo 7 do sexo masculino. Dentre as mães, com idade entre 22 e 43 anos, havia quatro com nível superior de escolaridade, uma com ensino médio completo, três com ensino fundamental completo e duas com ensino fundamental incompleto.

\section{Instrumentos}

Foram utilizados três instrumentos para a coleta de dados. Para a avaliação da interação mãe-bebê foi utilizado o Protocolo de Observação da Interação Mãe-Bebê 0-6 meses (POIMB 0-6) de Schermann (2007). O instrumento contém 21 itens, sendo 12 referentes ao comportamento da mãe, 8 referentes ao comportamento da criança, e um referente à sintonia da interação, que corresponde a uma avaliação geral dos comportamentos da díade. Os itens são avaliados em uma escala Likert de 5 pontos, em que escores mais altos equivalem à maior frequência ou a um desempenho mais satisfatório. Na situação de observação estavam disponíveis bichinhos de borracha, móbiles e chocalhos. Uma filmadora e fitas foram utilizadas para o registro das interações.

Para a avaliação da ansiedade materna foi utilizada a Escala de Ansiedade de Hamilton (HAMA) (Hamilton, 2000), que é autoadministrada. Compreende 14 itens distribuídos em dois grupos, cada qual com 7 itens, sendo o primeiro grupo relacionado a sintomas de humor ansioso, e o segundo relacionado a sintomas físicos de ansiedade, possibi- 
litando a obtenção dos escores parciais, além do total. Cada item é avaliado através de uma escala de Likert de 5 pontos, que vai da ausência (0) até a alta intensidade dos sintomas (4). A pontuação máxima é de 56 pontos, considerando de 0 a 17 pontos, sem ansiedade; de 18 a 24 pontos, com ansiedade leve; de 25 a 29 pontos, com ansiedade severa e, mais que 30 pontos, com ansiedade severa (Hartman Junior et al., 2012).

Para a avaliação do estresse foi utilizado o Inventário de Sintomas de Stress para Adultos de Lipp (ISSL) (Lipp, 2000). Esse instrumento é composto por três quadros de respostas com sintomas comuns na manifestação do estresse, sendo o primeiro relativo à fase de alerta; o segundo, à fase de resistência e quase exaustão; e o terceiro à fase de exaustão. Todos os quadros possuem a descrição dos sintomas somáticos e psicológicos, permitindo indicar a área predominante de manifestação de sintomas.

\section{Procedimentos}

O estudo foi aprovado pelo Comitê de Ética e Pesquisa da Universidade de São Paulo, conforme Resolução 196/96 do CNS (Processo n 091/2010). Aprovado o projeto, todas as mães com bebês internados com a SR foram contatadas individualmente e convidadas a participar, tendo sido explicitados os objetivos da pesquisa. Após os esclarecimentos solicitados, o Termo de Consentimento Livre e Esclarecido foi assinado por 10 das 13 mães convidadas.

A coleta de dados foi realizada na Unidade de Cuidados Especiais do HRAC/USP. A filmagem da interação mãe-bebê foi realizada em horário previamente combinado, dependendo das condições físicas da criança (estar acordada, alimentada, trocada). No ambiente foram disponibilizados brinquedos, mas não foi sugerida às mães a sua utilização. A filmagem mãe-bebê durou 10 minutos pra cada díade, sendo as mães orientadas a ficar com o bebê, para que a filmagem pudesse ser realizada. Após a filmagem, a mãe respondia à HAM-A e ao ISSL.

As interações mães-bebês, obtidas a partir 134 das filmagens, foram analisadas por dois avaliadores independentes, de acordo com as categorias estabelecidas pelo POIMB 0-6 (Schermann, 2007). Considerou-se tanto os comportamentos individuais de cada membro da díade quanto a avaliação geral da qualidade de sintonia da interação: nenhuma, pouca, moderada, muita e sempre. $\mathrm{O}$ acordo entre os avaliadores foi de $100 \%$ depois de dirimidas as divergências e reanalisadas as observações.

Os dados obtidos com a aplicação dos instrumentos HAM-A e ISSL foram analisados de acordo com o manual e organizados em tabelas descritivas. Os resultados obtidos nos instrumentos de avaliação de ansiedade (HAM-A) e estresse (ISSL) e os de sintonia de interação mãe-bebê (situação de observação) foram organizados em tabelas. Para analisar as correlações entre variáveis utilizou-se o Coeficiente de Correlação de Spearman, considerando-se nível de significância de $5 \%$.

\section{Resultados e Discussão}

De acordo com a avaliação de ansiedade conduzida, todas as mães apresentaram-se ansiosas, em diferentes graus: cinco apresentavam ansiedade severa; uma, moderada; e quatro, leve. Em relação à avaliação do estresse, oito mães apresentaram quadro de estresse, sendo uma na fase de exaustão, três na de quase exaustão, e quatro na de resistência. Todas essas mães apresentaram prevalência de sintomas psicológicos. Esses dados podem ser observados na Tabela 1.

Relacionando os níveis de ansiedade e estresse, nota-se que, das cinco mães que apresentaram ansiedade severa, uma estava na fase pior de estresse, a de exaustão, e três na de quase exaustão. Análise estatística apontou correlação positiva entre o nível de ansiedade e de estresse (0,0041, $R=0,815)$, ou seja, as mães mais ansiosas eram também as mais estressadas. Esse dado parece indicar uma relação positiva também entre esses constructos, ainda que já seja possível avaliar separadamente o estresse. Padovani et al. (2004) também constataram que no contexto de hospitalização torna-se comum identificar, nas mães que acompanham o tratamento de bebês de risco, altos níveis de ansiedade e sentimentos exacerbados de tristeza e melancolia, diante dessa situação de conflito e 
Tabela 1

Descrição dos níveis de ansiedade das mães, fases de estresse e manifestação de sintomas predominantes

\begin{tabular}{|c|c|c|c|c|}
\hline \multirow{2}{*}{ Mães } & \multicolumn{2}{|c|}{ HAM - A } & \multicolumn{2}{|c|}{ ISSL } \\
\hline & Nível de ansiedade & Sintomas predominantes & Fases de estresse & Sintomas predominantes \\
\hline 1 & Leve & Psicológicos & - & - \\
\hline 2 & Leve & Psicológicos & Resistência & Psicológicos \\
\hline 3 & Leve & Psicológicos & - & - \\
\hline 4 & Leve & Físicos & Resistência & Psicológicos \\
\hline 5 & Moderada & Psicológicos & Resistência & Psicológicos \\
\hline 6 & Severa & Psicológicos & Resistência & Psicológicos \\
\hline 7 & Severa & Psicológicos & Quase exaustão & Psicológicos \\
\hline 8 & Severa & Psicológicos & Quase exaustão & Psicológicos \\
\hline 9 & Severa & Psicológicos & Quase exaustão & Psicológicos \\
\hline 10 & Severa & Físicos & Exaustão & Psicológicos \\
\hline
\end{tabular}

Nota: ISSL: Inventário de Sintomas de Stress para Adultos de Lipp; HAM-A: Escala de Ansiedade de Hamilton.

estresse. Segundo Carvalho (2005), a presença de sintomas de ansiedade e depressão pode significar situação de risco para a relação mãe-bebê, comprometendo a qualidade dos cuidados maternos. Fraga et al. (2008) também encontraram evidências sobre as repercussões emocionais negativas nas crianças, em função dos quadros de ansiedade materna.

As médias dos comportamentos categorizados na situação de observação, emitidos pelas mães e pelos bebês, encontram-se na Tabela 2 . Verifica-se que as mães interagem mais $(p=0,0039)$, o que era esperado em função da imaturidade dos bebês. Entre os comportamentos mais frequentes das mães observou-se a presença de comportamentos favoráveis, mas também desfavoráveis à interação. Entre os favoráveis, os mais frequentes foram: a atenção da mãe com a criança $(4,3)$, ou seja, se a mãe está atenta ao filho(a); a quantidade de contato corporal da mãe com o filho(a) durante o período da observação $(4,2)$ e a quantidade de contato visual ou tentativa de contato visual da mãe para com o bebê $(4,2)$. Entre as desfavoráveis, observou-se a expressão de afeto negativo $(4,6)$, que envolve as expressões verbais e não verbais de frustração, impaciência, desgosto e irritabilidade.

Também entre os bebês observaram-se as duas classes de comportamentos: favoráveis e desfavoráveis à interação, ainda que em frequência baixa. Entre os favoráveis, foram observados: o bom humor $(2,8)$, caracterizado por comportamentos que indicam sinais de prazer, como o sorrir, o balbuciar ou a excitação corporal; o olhar para o rosto da mãe $(2,6)$; e a intensidade com que a criança responde à tentativa de estabelecer comunicação por parte da mãe $(2,6)$, isto é, se a criança reage olhando, mexendo-se, vocalizando, sorrindo, através de expressões da face ou movimentação corporal, bem como a resposta aos estímulos comunicativos da mãe, se a criança a responde a eles constantemente de maneira positiva. Entre os desfavoráveis, o comportamento de choro ou choramingo foi o mais frequente $(4,0)$.

Outro dado analisado foi a sintonia de interação da díade, ou seja, a quantidade e qualidade da interação entre a mãe e a criança, em termos de comunicação e contato. Os resultados indicaram que quase metade das díades (quatro) foi classificada como apresentando pouca qualidade de interação, enquanto três outras apresentaram sintonia tida como moderada, indicando que mãe e filho(a) demonstraram interação positiva em metade do tempo da filmagem. Apenas uma díade apresentou a classificação de "ótima sintonia de interação", mostrando relações positivas constantemente, com boa qualidade, ao passo que duas outras apresentaram muita sintonia, indicando interação positiva na maior parte do tempo. Assim, os dados do estudo indicam que a maior parte das interações foi julgada como mediana ou pobre, diferentemente dos dados encontrados por Seidl-de-Moura et al. (2008) com bebês saudáveis, em torno de um mês de idade. 
Tabela 2

Média de ocorrência dos comportamentos maternos e dos bebês em situação de observação

\begin{tabular}{|c|c|}
\hline Categorias & Média dos comportamentos das Mães \\
\hline Comunicação verbal & 4,2 \\
\hline Contato visual ou tentativa de contato & 4,2 \\
\hline Contato corporal & 4,3 \\
\hline Expressão de afeto positivo & 3,9 \\
\hline Eficiência para consolar o bebê quando chora & 1,9 \\
\hline Reação da mãe ao choro, choramingo ou agitação do bebê & 2,7 \\
\hline Resposta ao comportamento social do bebê & 3,9 \\
\hline Intensidade da resposta positiva da mãe ao comportamento social do bebê & 3,9 \\
\hline Expressão de afeto negativo & 4,6 \\
\hline Sensitividade materna & 3,7 \\
\hline Atenção da mãe com do bebê & 4,3 \\
\hline Intrusividade & 3,4 \\
\hline \multirow[t]{2}{*}{ Média Total } & 3,7 \\
\hline & Média dos comportamentos dos bebês \\
\hline Quantidade de olhares para o rosto materno & 2,6 \\
\hline Resposta às comunicações & 2,3 \\
\hline Intensidade de resposta positiva & 2,6 \\
\hline Contato físico e/ou verbal & 1,9 \\
\hline Quantidade de sorriso & 1,3 \\
\hline Bom humor & 2,8 \\
\hline Quantidade de vocalização & 1,4 \\
\hline Quantidade de choro e choramingo & 4,0 \\
\hline Média Total & 2,3 \\
\hline
\end{tabular}

Analisando os comportamentos de interação das mães com os dos bebês, observou-se correlação positiva entre eles ( $p=0,00027, R=0,908)$, isto é, quanto mais interativa é a mãe, mais também é o bebê.

Segundo Bowlby (1990), uma relação mãe-bebê adequada não depende apenas dos comportamentos maternos, na medida em que mãe e bebê se autoestimulam a partir do contato que estabelecem. Seis bebês do estudo apresentaram pouco ou nenhum contato visual e pouca verbalização; enfim, poucas respostas às tentativas de comunicação por parte das mães. Por outro lado, três mães agiram de modo muito intrusivo, tentando chamar a atenção dos bebês de forma exagerada e contínua, sem se ater às respostas deles. Mães agitadas e ansiosas tendem a ser mais intrusivas, fazendo com que o excesso de estimulação por parte delas, somado ao excesso de estimulação encontrado no ambiente de UTIN - tais como excesso de ruído, luz ininterrupta, manuseios em diferentes posições e procedimentos dolorosos -, produzam sensações desconfortáveis ao bebê, podendo causar reações de angústia e "respostas de retirada" tais como caretas e olhos fechados com força (Lira, 2004; Fraga et al., 2008).

Por meio da teoria do apego, Bowlby (1990) e Ainsworth et al. (1978) vêm destacando que é fundamental que a mãe se mostre atenta e sensível aos sinais do bebê, sendo capaz de colocar-se no lugar dele. A sensibilidade materna permite que a mãe responda adequadamente às necessidades de comunicação do bebê, promovendo uma base segura para seu desenvolvimento emocional (Ainsworth et al., 1978; Alfaya \& Schermann, 2005).

A análise da relação entre a qualidade da interação e da sintonia na interação mãe-bebê e a ansiedade e o stress materno estão apresentados na Tabela 3.

Verifica-se que, nas quatro díades classificadas como tendo "pouca" sintonia de interação, os níveis de ansiedade maternos eram severos, assim como foram encontrados níveis de stress de quase exaustão ou exaustão. Nas duas díades considera- 
Tabela 3

Média da ocorrência de comportamentos maternos e infantis na situação de observação, sintonia de interação das díades e classificação dos níveis de ansiedade e estresse maternos

\begin{tabular}{|c|c|c|c|c|c|}
\hline$N^{\circ}$ & Mães & Bebês & Sintonia da Interação & Ansiedade & Estresse \\
\hline 1 & 3,4 & 3,1 & Moderada & Leve & - \\
\hline 2 & 4,9 & 3,8 & Muita & Leve & Resistência \\
\hline 3 & 4,9 & 4,0 & Muita & Leve & - \\
\hline 4 & 3,6 & 3,4 & Moderada & Leve & Resistência \\
\hline 5 & 4,1 & 3,9 & Ótima & Moderada & Resistência \\
\hline 6 & 4,1 & 3,5 & Moderada & Severa & Resistência \\
\hline 7 & 2,8 & 2,3 & Pouca & Severa & Quase exaustão \\
\hline 8 & 3,3 & 2,8 & Pouca & Severa & Quase exaustão \\
\hline 9 & 3,7 & 2,9 & Pouca & Severa & Quase exaustão \\
\hline 10 & 3,8 & 3,2 & Pouca & Severa & Exaustão \\
\hline
\end{tabular}

das com "muita" sintonia de interação, o nível de ansiedade era leve, e o estresse de uma mãe foi classificado como de resistência, ou seja, ela ainda estava conseguindo exercer algum tipo de controle sobre a situação vivenciada. Pode-se constatar também que, na única díade com ótima sintonia de interação, a mãe apresentava ansiedade moderada e também estava na fase de resistência do estresse, ou seja, ela não era a que tinha menos sintomas emocionais, apesar de não estar entre os casos mais "graves". Já quando a sintonia foi classificada como moderada, em três díades, o nível de ansiedade variou de leve a severo, e o estresse ficou na fase de resistência ou nem foi classificado, isto é, praticamente não havia sintomas de estresse.

Em uma análise considerando a relação entre o nível de ansiedade e o nível de sintonia da interação, observou-se uma relação inversa estatisticamente significativa: quanto maior a ansiedade, menor a sintonia de interação $(p=0,0163, R=0,731)$. Em relação ao estresse também se observou correlação negativa ( $p=0,0078, R=0,78)$ : quanto maior o estresse, menor a sintonia de interação.

A ansiedade experimentada pelas mães possivelmente pode estar atrelada a um estado emocional temporário, marcado por sentimentos desagradáveis de tensão e apreensão, reativos à situação de internação do bebê (Padovani et al., 2004). Por outro lado, Castro e Piccinini (2004) alertam que as crianças internadas geralmente passam por frustrações agudas e intensos sofrimentos físicos e emocionais com a hospitalização, cirurgia e outros proce- dimentos invasivos, afetando o processo de construção da interação com a mãe.

Nos casos em que a sintonia de interação foi avaliada como positiva (ótima ou muita), pode-se inferir que essas mães estavam em melhores condições emocionais dos que aquelas que apresentavam níveis superiores de ansiedade e de stress e que, provavelmente, elas apresentavam mais condições de ajudarem o filho a enfrentar a hospitalização e as condições físicas desconfortáveis. Todavia, é importante destacar que pode haver exceções. Mães com problemas emocionais podem apresentar boa sintonia com seus bebês, o que indica que outros fatores também podem estar envolvidos na interação. Feijó (1998) destaca que, quando os genitores são sensíveis e oferecem respostas a seus bebês, estão capacitando-os a seguir um caminho saudável, ao passo que, quando são insensíveis, não responsivos e rejeitadores, é possível que seus filhos sigam por um caminho incompatível ao caminho saudável nas situações adversas.

Os dados indicam a possibilidade de se associar a ansiedade e o estresse ao comprometimento da relação mãe-bebê, sugerindo que a mãe com altos níveis de ansiedade e estresse pode não desenvolver de uma maneira satisfatória a sensibilidade materna. Baldini e Krebs (2005) ressaltam que pais que não conseguem passar por essa fase de forma adequada acabam por encontrar barreiras físicas e psicológicas internas, fazendo com que a ligação com a criança seja prejudicada. 
Mães que apresentam maior nível de ansiedade e estresse necessitam, portanto, de mais atenção dos profissionais para se adaptar melhor à situação que vêm enfrentando e, também, para ter condição de minimizar o sofrimento do filho (Baldini \& Krebs, 2005; Brum \& Scherman, 2004). A situação de risco que envolve o bebê necessitado de cuidados intensivos, bem como o tempo de sua internação, podem despertar na mãe sentimentos de intensa culpa e profunda tristeza, prejudicando o surgimento do sentimento de competência materna e, consequentemente, da sensibilidade materna (Alfaya \& Schermann, 2005).

Brum e Shermann (2004) destacam que a conexão emocional da díade e o comportamento de apego podem ficar comprometidos, e que muitas vezes o cuidado que a mãe pode oferecer não é suficiente às necessidades psicológicas da criança para o estabelecimento de uma relação adequada. Durante a observação verificou-se que mães com menores níveis de ansiedade e estresse estavam mais atentas às necessidades do bebê, apresentando melhor interação e melhor capacidade de adaptação à situação, tal como descrito por Seidl de Moura et al. (2008).

Além disso, a permanência prolongada no espaço hospitalar, adicionada a vivência de situação de risco, impede a mãe de cuidar de si própria e atender suas necessidades de alimentação, repouso e sono, manifestando sinais evidentes de cansaço físico e mental (Lira, 2004; Castro \& Piccinini, 2004; Mendes, 2007). Correia e Linhares (2007) destacam que as mudanças físicas ocorridas durante e após a gravidez, por si sós, já podem provocar instabilidade emocional na mulher. Esses períodos de internação logo após a gestação envolvem grandes transformações nas mães, não só do ponto de vista fisiológico, mas também do ponto de vista psíquico. As contribuições da literatura ajudam a compreender a possível relação entre altos níveis de estresse e ansiedade maternos e a dificuldade no estabelecimento da relação mãe-bebê satisfatória.

A presença de sintomas de ansiedade e estresse nas mães de bebês com SR contribui para que elas se sintam instáveis emocionalmente, 138 principalmente nos casos em que a indicação cirúrgica é uma possibilidade. Correia e Linhares (2007) afirmam que, quando ocorrem tais desordens de saúde mental materna, elas podem causar efeitos prejudiciais ao desenvolvimento infantil. Há evidências de que efeitos dos quadros de ansiedade materna repercutem emocionalmente tanto na mãe quanto na criança (Perosa, Silveira, \& Canavez, 2008). Desse modo, os sintomas emocionais maternos após o nascimento do bebê têm sido apontados como fator de risco para o desenvolvimento da criança (Fraga et al., 2008).

\section{Conclusão}

O objetivo deste estudo foi investigar a relação entre ansiedade e estresse de mães de bebês internados com SR, e a interação estabelecida entre elas e seus bebês. Foi possível verificar que há relação entre a saúde mental materna e o modo de relação estabelecido pela mãe com seu bebê, ou seja, quanto mais elevados foram os níveis de ansiedade e estresse experienciados pelas mães, mais incipiente se classificou a sintonia de sua interação com o bebê.

A investigação acerca da relação entre a saúde mental materna e a qualidade de interação mãe-bebê é considerada relevante para a prática da Psicologia Clínica, visto que durante a internação hospitalar o foco do atendimento clínico é somente o bebê e seu desenvolvimento, não se considerando a influência do estado emocional da mãe na recuperação e desenvolvimento da criança. Porém, na medida em que se introduzem práticas que incluem a mãe, verifica-se que emergem conteúdos emocionais. Brum e Schermann (2004) ressaltam que a mãe deve ser acolhida em seu processo de luto pela perda do bebê idealizado, de forma que isso possa ser verbalizado e aceito pela equipe hospitalar. De forma gradativa, a mãe deve receber as informações sobre as capacidades do recém-nascido, participando ativamente de seu cuidado, estimulando, assim, a interação e favorecendo a sensibilidade materna no processo de formação do vínculo afetivo.

$\mathrm{O}$ presente estudo avalia a ansiedade materna utilizando um instrumento pouco frequente 
em estudos com essa população, mas que se mostrou eficiente ao identificar níveis críticos de ansiedade, semelhantes aos encontrados em outros estudos da área. Ainda inova ao avaliar o estresse materno, uma vez que ainda são incipientes os estudos que o avaliam, muito embora a situação de hospitalização seja considerada um evento potencialmente estressor. Ainda que com uma amostra reduzida, dada a especificidade do quadro (bebês com SR), os resultados sinalizam a presença de níveis de ansiedade e estresse preocupantes.

Outro desafio foi a filmagem da interação mãe-bebê em situação hospitalar, considerando toda a rotina de cuidados característicos desse tipo de contexto. Todavia, ainda que se observe uma alteração possível no comportamento da mãe ciente de que está sendo observada, a observação continente do comportamento do bebê compensa esse viés. É um procedimento que tem-se mostrado eficiente para a avaliação da interação mãe-bebê, amparada por um protocolo de análise refinado, como se mostrou o POIMB.

Ainda que os resultados se refiram a uma amostra relativamente pequena, os dados apontam a necessidade de propor alternativas de atenção aos cuidadores, os quais sofrem no processo de internação de um recém-nascido que exija cuidados intensivos. Identificar aspectos de sua saúde mental e propor serviços específicos é uma forma de auxiliá-los a lidar com conteúdos emocionais que podem dificultar a boa interação com o bebê. Nesse aspecto, é fundamental o preparo da equipe de saúde, que pode auxiliar no desenvolvimento psíquico da díade mãe-bebê.

\section{Referências}

Ainsworth, M., Blehar, M., Waters, E., \& Wall, S. (1978). Patterns of attachment: A psychological study of the strange situation. Hillsdale: Erlbaum.

Alfaya, C., \& Schermann, L. B. (2005). Sensibilidade e aleitamento materno em díades com recém-nascidos de risco. Estudos de Psicologia, 10, 279-285.

Andrade, L. H. S. G., \& Gorenstein, C. (1998) Aspectos gerais das escalas de ansiedade. Revista de Psiquiatria Clínica, 25(6), 285-290.
Andreani, G., Custódio, Z. A. O., \& Crepaldi, M. A. (2006). Tecendo as redes de apoio na prematuridade. Aletheia, 24, 115-126.

Baldini, S. M., \& Krebs V. L. J. (2005). Reações psicológicas nos pais de recém-nascidos internados em unidade de terapia intensiva. Pediatria Moderna. 36, 242-246.

Bowlby, J. (1990). Apego e perda. Apego (Vol. 1). São Paulo: Martins Fontes.

Brum, E. H. M., \& Schermann, L. (2004). Vínculos iniciais e desenvolvimento infantil: abordagem teórica em situação de nascimento de risco. Ciência e Saúde Coletiva, 9(2), 457-467.

Camarneiro, A. P. F., Alves, C. A. N., Ferreira, A. P. C., \& Gomes, A. I. F. (2009). Interação mãe-bebê prematuro numa Unidade de Cuidados Intensivos Neonatais. Acta Pediátrica Portuguesa, 40(2), 53-57.

Carvalho, A. E. V. (2005). Indicadores emocionais maternos e intervenção psicológica durante a internação do bebe pré-termo em UTI Neonatal (Tese de doutorado não-publicada). Universidade de São Paulo, Ribeirão Preto.

Castro, E. K., \& Picinini, C. A. (2004). A experiência de maternidade de mães de crianças com e sem doenças crônicas no segundo ano de vida. Estudos de Psicologia (Campinas), 9(1), 89-99. doi:

Correia, L. L., \& Linhares, M. B. M. (2007). Ansiedade materna nos períodos pré e pós-natal: revisão da literatura. Revista Latinoamericana de Enfermagem, 15(4), 677-683.

Dantas, M. M. C., Araújo, P. C. B., Paulino, D. S., \& Maia, E. M. C. (2012). Avaliação do apoio social e de sintomas depressivos em mães de bebês prematuros hospitalizados. Psicologia em Revista, 18(1), 90-106.

Feijó, O. L. (1998). O bebê pré-termo: intervenção precoce visando à melhoria da interação mãe-bebê (Dissertação de mestrado não-publicada). Universidade Federal do Rio Grande do Sul, Porto Alegre.

Fraga, D. A., Linhares, M. B. M., Carvalho, A. E. V., \& Martinez, F. E. (2008). Desenvolvimento de bebês prematuros relacionado a variáveis neonatais e maternas. Psicologia em Estudo, 13(2), 335-344.

Fuzza, R. F., \& Abuabara, A. (2010). Sequência de Pierre Robin no recém-nascido: relato de caso. Pediatria, 32(3), 231-235.

Hamilton, M. (2000). Escala de avaliação de ansiedade HAM-A. In C. Gorenstein, L. H. S. G. Andrade, \& A. W. Zuardi. Escalas de avaliação clínica em Psiquiatria e Psicofarmacologia (pp.89-95). São Paulo: Lemos Editorial.

Hartman Junior, J. A. S., Gomes, G. C. G., Januário, A. C. S., Silva, R. A., Sougey, E. B., Peregrino, A., \& Bastos, O. (2012) Estados ansiosos e depressivos de idosas institucionalizadas. Revista de Neurobiologia, 77(1,2), 147-153. 
lungano, E. M. (2009). A relação entre a mãe e o bebê prematuro internado em UTI neonatal. Psicologia em Pediatria, 45(1), 26-30.

Linhares, M. B. M., Carvalho, A. E. V., Machado, C., \& Martinez, F. E. (2003). Desenvolvimento de bebês nascidos pré-termo no primeiro ano de vida. Paidéia, 13(25), 57-72.

Lipp, M. E. N. (2000). Inventário de sintomas de stress para adultos. São Paulo: Casa do Psicólogo.

Lipp, M. N., \& Romano, A. S. F. (1987). O stress infantil. Estudos de Psicologia, 4, 42-54.

Lira, M. M. F. L. (2004). Atendimento Humanizado em Unidade de Terapia Intensiva Neonatal. In Assistência ao Recém-Nascido de Risco. Brasília: Porfiro.

Marques, I. L., Souza, T. V., Barbieri, M. A., \& Bettiol, H. (2005). Sequência de Robin: protocolo único de tratamento. Jornal de Pediatria, 81, 14-22.

Mendes, E. A. (2007). Experiência com um grupo de mães em um hospital considerando o vínculo materno. Psicoscopio, 6(3), 20-28.

Padovani, F. H. P., Linhares, M. B. M., Carvalho, A. E. V., Duarte, G., \& Martinez, F. E. (2004). Avaliação de sintomas de ansiedade e depressão em mães de neonatos pré-termos durante e após hospitalização em UTI-neonatal. Revista Brasileira de Psiquiatria, 26(4), 251-254.

Perosa, G. B., Silveira, F. C. P., \& Canavez, I. C. (2008). Ansiedade e depressão de mães de recém-nascidos com malformações visíveis. Psicologia: Teoria e Pesquisa, 24(1), 29-36.

Reichert, A. P. S, Lins, R. N. P., \& Collet, N. (2007). Humanização do cuidado em UTI Neonatal. Revista Eletrônica de Enfermagem, 9(1), 200-213. Recuperado em agosto 13, 2012, de http://fen.ufg.br.Qrevista/v9/ n1/v9n1a16.htm

Rey, G. J. F. D. (2005). Quando a ansiedade torna-se uma doença? Revisa Integração, 43, 379-382.

Sato F. R. L., Setten, K. C., Sverzut, A. T., Moraes, M., Moreira, R. W. F. (2007). Sequência de Pierre Robin: Etiopatogenia, características clínicas e formas de tratamento. Revista Portuguesa de Estomatologia, Medicina Dentaria e Cirurgia Maxilofacial, 48(3), 161-166.

Scochi C. G. S., Kokuday, M. L. P., Riul, M. J. S., Rossanez, L. S. S., Fonseca, L. M. M., \& Leite, A. M. (2003). Incentivando o vínculo mãe-filho em situação de prematuridade: as intervenções da enfermagem no Hospital das Clínicas de Ribeirão Preto. Revista Latinoamericana de Enfermagem, 1(4), 539-544.

Schermann, L. (2007). Avaliação quantitativa e qualitativa da interaçãa mãe-bebê. In C. A. Piccinini, \& M. L. S. Moura. (Orgs.), Observando a interação pais-bebê-criança (pp.155-175). São Paulo: Casa do Psicólogo.

Seild-de-Moura, M. L., Ribas, A. F. P., Seabra, K. C., Pessoa, L. F., Nogueira, S. E., Mendes, D. M. L. F., ..., Vicente, C. C. (2008). Interações mãe-bebê de um e cinco meses: aspectos afetivos, complexidade e sistemas parentais predominantes. Psicologia Reflexão Crítica, 21(1), 66-73.

Souza, J. A. (2005). A formação do vínculo afetivo: a questão do apego. Revista Técnica do IPEP, 5(1/2), 81-98.

Wust, G. G., \& Viera, C.S. (2011). O relacionamento mãe-bebê pré-termo após alta hospitalar. Cogitare Enfermagem, 16(2), 311-318.

Recebido: outubro 25, 2012

Versão final: maio 8, 2013

Aprovado: maio 17, 2013 